\title{
SOBRE UN CASO DE TUBERCULOSIS INTRAUTERINA
}

\author{
Drs. JULIO ESPINOZA S., HUMBERTO RECCHIONE F. \\ y GUILLERMO GARCIA N. \\ Instituto de Anatomía Patológica y Cátedra Extraordinaria de Pediacria del Prof. A Sreeger. \\ Hospital "Manuel Arriarán".
}

Victor L. B. Peso 1.680 grs. Talla 40 cms. C. C, 29. Tórax $26.5 \mathrm{cms}$.

El presente ciso se réfiere a la observación de un prematuro nacido en la Macernidad de San Borja el 30-I-53. a las 4.55 hor.1s.

Visto por el médico puericultor a las 9 horas del mismo día. y por declaración de la madre, de entontrarse enferma diel pulmón. sin precisar mayores datos. se ordenó su inmediata separación de la madre. En consecuencid este giño estuvo junto a su madte 4 boras 5 minutos. Se hizo trasladar al día siguiente a este Hospital, al Servicio de Medicina. Por una omi. sión de informes proporcionados por maternidad no se consignó la probabilidad de tuberculosis de la madee. Er el Servicio fué vislo por el Dr. Aranda quicr anotó los datos conzernientes al examen del niño $y$ de jó las indicacioncs de rigor para un prematuro. En su evolución no hubo nada especial anotado, hasta el $17^{\prime 2}$ dia en que apatecicton crisis de asfixia que se pensó que podian set por aspiración de leche. Pero se observaron en la curva térmica, algunas alzas que fueron arribuidas a calentamiento de la incubadora. Posteriormente evoluciono casi hipotérmico. Las crisis asficticas comenzaron a tepetirse con cierta frecuencia. encontríndose en su examen fisico sólo zonas de atelectasia derecha y cierro temblot fibriltar de sus extremidades que aconsejaton una puncion lumbar que resultó negativa. Las ctisis de asfixia se hicieron más frecuentes y gtaves, acompañadas de cianosis y de un progresiso mal aperito que conđicionaton una alimentución defectuosa, que se resolvió en parte con sonda gástrica.

En el último examen practicodo se anotó: disnea. intermitencia respiratoria, ¿zonas atelectásieas?, tercio medio dercho. En consecuencis, no habia elementos suficientes para plantear ningún otro diagnóstico que e) de prematurez.

El tratamiento se hizo de acuerdo con las normas Lisuales para estos ajĩos, agregando el uso de penicir lina, dado el cuadro de atelectasia que prescntaba, y complementado con oxigeno, veriazol, etc. El niño falleció en una de sus crisis asficticas, a los 26 dias de edad y fuć cnviado a Anatomia Patológica con el diagnóstico de Prematurez.

En la necropsia ( $N^{0} 119 / 53$ se encontraron los pulmones, tanto en la superficie pleutal como en el espesor de ellos. sembrados de gránulos de color blanquecino-amarillento, del tamaño de una cabeza de alfiler y mayores. En la tegión paratraqueal inferior de- recha y cara anterior del hilio pulmonar izquierdo babia sendos ganglios pequeños, poco mayores que uns lenteji, de consistencia firme y al corte totalmente caseosos. El higado en su cara superior, presentiba tres fequeños gránulos de color blanquecino y menores que la cabeza de un alfifer. El bazo tambien mostrabu en su cata externa un pequeño grínulo de igual aspecto al descrito en el higado. El bígado y bazo ( 70 y 10 grs. respectivamenre), esraban ligera. mente aumentados de tumaño.

En el examen microscópico del pulmón detecho (Jóbulo superior) se encontraron mültiples y pequeños focos necróticos a cupo rivel sólo se observaban restos de núcleos y escasas cólulas redondas pequeñas. Los alvéolos vecinos se presentaban parcialmente atelectásicos y ocupados por grandes células oroídeas. con abundante citoplasma eosínófilo y núcleos redondeados con finos gránulos cromácicos; algunas de est.ls células contenían pigmento café-smarillento en forma de finos grinulos. La coloración específica del bacilo de Koch fuć intensamente positiva en los focos necrótiens discritos. Estos focos nectóticos estaban ubicados tanto en los alvéolos como en conductos alveolates e intersticio (Microfotografís $N^{\prime \prime} \perp$ y 2). Examinado al microscopio el ganglio paratraqueal, se encontró casi toralmente octpado por focos necróticos de idéntico aspecto a los descritas en el pulmón. persistiendo sólo en su períferia algunos nódulos linfocitarios (Microfotografia $N^{0} 3$ ). Tanto en el palmón como en este ganglio no se obsicyó ninguna formación de tuberculosis productiva.

\section{COMENTARIO}

De acuerdo con lo expuesto, se trata de un prematuro, hijo de madre tuberculosa y que convivió con ella alrededor de 4 horas. Posteriormente hemos sabido que la madre padece de tuberculosis pulmonar cavitaria bilateral, tuberculosis laríngea e intestinal y que aún vive (Noviembre, 1953).

Los hallazgos anátomo-patológicos del niño corresponden a lesiones de tipo exudativo, diseminadas en los pulmones, higado, bazo y ganglios paratraqueal derecho y del hilio pulmonar izquierdo, lesiones controladas microscópicamente en el pulmón y ganglios. Las lesiones de estos últimos órganos (pulmón y ganglios), exu- 


\section{MICROIOTOGRAFIA N"}

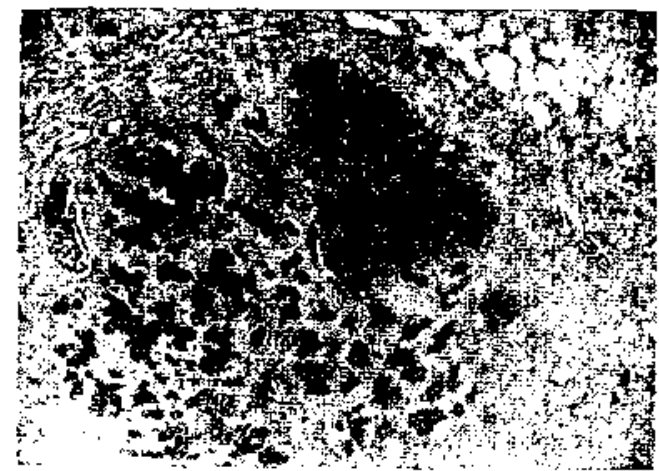

$\mathrm{X}$ 20. Hematoxilina-Eosina. Pulmón. Múltiples pequeños focos exudativo-caseosos que abectan a alviolos. conductos alveolares $\mathfrak{e}$ intersticic.

dativas y con gran tendencia a la necrosis, aparentaban corresponder a un proceso de tiempo de evolución análoga, es decir, no se apreciaba un foco que pudiera considerarse de mayor antigüedad. En el aparato digestivo y meninges no se apreciaron lesiones macroscópicas especím ficas o inespecificas.

Creemos que en este caso estamos frente a una tuberculosis intrauterina por las siguientes razones:

$1^{\circ}$ Niño separado de su madre pocas horas (4) después del nacimiento, madre que revelaba graves lesiones pulmonares tuberculosas.

$2^{\circ}$ Niño con lesiones tuberculosas generalizadas, de tipo exudativo y con gran tendencia a la caseificación.

$3^{\circ}$ Ausencia de un foco tuberculoso de mayor antigüedad.

\section{MICROFOTOGRAFIA $N^{*} 2$}

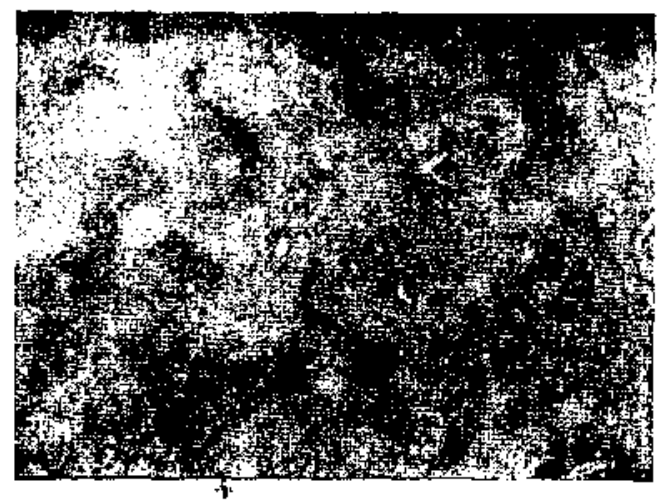

$X$ 70. Hematoxilina-Eosina. Ganglio Patatraqueal. Extensas zonas exudativo-caseosas.

\section{MICROFOTOGRAIIA NO3}

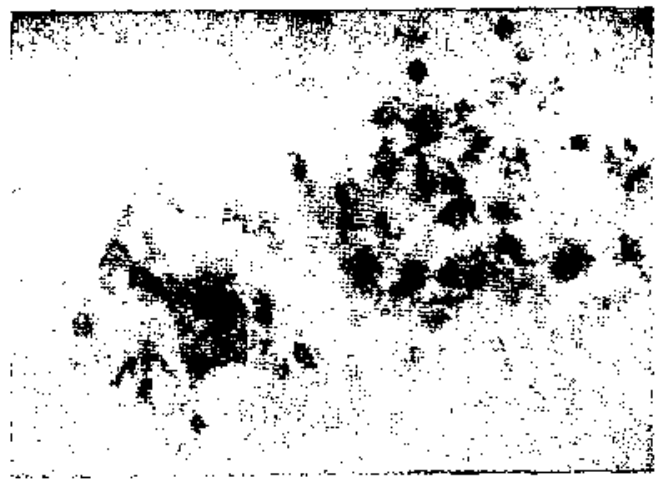

$X$ 90. Coloración especifica para bacilos de Koch. Pulmón.

$4^{\text {y }}$ Muerte del niño alrededor de Ia $3^{a}$ semana (26 días).

Evidentemente, la tuberculosis intrauterina es un hecho raro, pero existen ejemplos indubitables de esta afección, tales como los descritos por Siegel, correspondiente a 38 casos separados de la literatura $y$ en los que se aceptó la evidencia de una infección intrauterina y 11 casos dados a conocer por Waissmann. Entre nosotros han sido publicados hasta la fecha 7 casos: 1 de Ariztía e Illanes; 2 de Scroggie y Kausel; 2 de Oscar Cereceda; 1 de Julio Schwarzenberg, Guzmán y Cousiño y 1 de Arturo de la Barra y Sergio Donoso.

Para explicar el contagio tuberculoso intrauterino, se han supuesto tres formas: $1^{\mathrm{a}}$ la sanguínea, vía vena umbilical, $2^{\mathrm{a}}$ la aspiración o deglución de líquido amniótico contaminado y $3^{2}$ la absorción de se. creciones o productos de lesiones tuberculosas del canal genital, durante el parto.

Según Rich, la infección por la placenta "resulta más frecuentemente por tuberculosis miliar que por una tuberculosis local del endometrio", aunque hay casos en que con compromiso importante de la placenta, el feto resulta libre de lesiones. Esta resistencía del feto al bacilo de Koch, reproducida experimentalmente por Vorwald, la explica este autor como debida, al menos en parte, a la baja tensión de oxígeno en la sangre fetal, sugestión apoyada por Rich y Follis mediante sus estudios sobre la inhibición de la tuberculosis en animales adultos mantenidos en un ambiente de oxígeno a baja tensión. 
En la infección sanguínea, via veno umbilical, los órganos más afectados son el hígado y los ganglios linfáticos perihepáticos $\mathrm{y}$ cuando se debe a aspiración de bacilos del líquido amniótico infectado o a endometrio infectado después de la rotura de las membranas, son, naturaimente, los pulmones, los más lesionados. Sin embargo, los pulmones pueden infectarse directamente desde la placenta por vía del conducto venoso de Arancio, rama de la vena umbilical que se vacia directamente en la cava, sin pasar por el higado. También conviene recordar el hecho que después del nacimiento, por el mayor aporte de oxigeno a los pulmones y las modificaciones que sufre la circulación a nivel del higado (abastecimiento sanguíneo en gran parte venoso y disminución de la cantidad de oxígeno en los sinusoides por el alto consumo que de él hacen las células hepáticas) puede observarse un desarrollo más intenso de las lesiones tuberculosas en los pulmones que en el hígado, pese a que este último fué el primitivamente infectado, vía vena umbilical. Finalmente, merece recordarse que entre los casos descritos por Siegel, hubo algunos en que los pulmones, predominantemente comprometidos, contenían focos neumóticos llenos de bacilos tuberculosos, lesiones atribuídas a aspiración de liquido amniótico infectado, pero que necesariamente pudieron no tener tal origen, ya que en forma experimental se ha observado que los bacilos pueden alcanzar el pulmón por vía sanguínea a partir de una localización subcutánea de la infección. A esto podemos agregar que Rich no ha encontrado un caso en la literatura en que la infección del amnios estuviera demostrada.

Volviendo a nuestro caso, creemos que las lesiones diseminadas del proceso tuberculoso exudativo caseoso, de predominio pulmonar, puedan haberse originado por vía sanguinea proveniente de la placenta, ya que la ausencia de lesiones en el aparato digestivo habla en contra de la deglución de líquido contaminado. Respecto a la edad de las lesiones encontradas, todas ellas, al parecer, establecidas simultáneamente, no es posible hacer un cálculo sobre la base de las lesiones encontradas. Sólo podemos recordar que es indispensable que transcurra cierto tiempo o período de incubación o prealérgico para que se produzca la caseificación, tiempo que para Wallgren oscila entre 19 y 5 dias. Un Iapso semejante en la aparición de la reacción tuberculinica se observó en los niños de Lübbeck, siendo el período más corto de 23 dias, fluctuando en general entre cuatro y diez semanas. Insistimos que en nuestro caso las lesiones diseminadas de tipo exudativo caseoso tienen un origen intrauterino, basados, además de las razones ya expuestas, en el carácter de los complejos primarios pulmonares descritos en los casos de Liibbeck, donde estos eran de tipo neumónico caseoso multiple en niños que recibieron la vacuna "per os", vía de contagio que habría que aceptar en el caso que comentamos, si supusiéramos una infección proveniente de su madre, ocurrida después del nacimiento $\mathrm{y}$ durante las 4 horas que permaneció con ella. Nos llama la atención, naturalmente, aunque no es un hecho excepcional, ya que en el último caso de la casuistica nacional también se observa, que la madre de este niño aún esta viva, tal vez por el tratamiento con antibióticos que pudo realizarse aunque tardíamente.

\section{RESUMEN}

Se relata la historia de un prematuro, hijo de tuberculosa, con la cual convivió 4 horas. Fallecido a los 26 días de edad, se encuentra en la necropsia una tuberculosis diseminada, de tipo exudativocaseoso, predominantemente pulmonar, la cual se cataloga de origen intrauterino. Se comenta la patogenia de esta forma rara de tuberculosis.

\section{SUMMARY}

The authors publish the case history of a premature, son of a tubercular mother with whom he lived four hours. Having died 26 days later, the autopsy showed a disseminated tuberculosis of an exudative caseous type, predominantly pulmonary, which is cataloged as of intrauterine origin. The pathogenesis of this rare form of tuberculosis is commented.

\section{BIBLIOGRAFÍA}

\footnotetext{
RJC.H, A. - Jatogenia le la tuberculosis. Fatitorial Alfa, B. Aires. 1946.

DE I,A BARHA, A $y$ JONOSO, L, - A propisito de un casc le tuberculasis congenia, Bol, de la Suc Chi. lena de Obstetricia y Gineculogia. VoI. XIV, Julio de 1949. \:4.

ASCHOFF. L. - Tratalo de Anatomia Patológita. Editoria! Labor, s. A. Barcelona-Ifadrid-B. Aires, 1934.
} 